\title{
Ergebnisorientiert und interessensgeleitet - Studien der Wirtschaftslobby zum GW-Unterricht
}

\author{
*anke.uhlenwinkel@aau.at, Alpen-Adria-Universität Klagenfurt \\ eingereicht am: 30.07.2017, akzeptiert am: 15.04.2018
}

Ende 2016 sah sich der GW-Unterricht in Österreich durch Vertreter ökonomischer Ansätze deutlich in Frage gestellt: GW-Lehrende seien nicht kompetent Wirtschaft zu unterrichten. Verschiedene empirische Studien versuchten diese Behauptung zu belegen. In diesem Beitrag werden zwei dieser Studien inhaltlich und methodisch auf ihre Validität geprüft. Hinsichtlich beider Aspekte wurden deutliche Mängel identifiziert. Darüber hinaus erweist sich der implizierte didaktische Ansatz als überholt. Im Ausblick wird das Potential des Ansatzes der Basiskonzepte für ein integriertes Fach GW diskutiert.

Keywords: Ökonomische Bildung, empirische Studien, lerntheoretische Ansätze, Unterrichtsplanung, Basiskonzepte

\section{Goal-oriented and interest-based - empirical studies of the business lobby relating to GW-education}

At the end of 2016, Austrian GW-Unterricht saw itself vehemently questioned by representatives of economic approaches. The main claim was, that GW-teachers were not fit to teach economics properly. Several empirical studies were conducted to prove this claim. This paper questions the validity of two of these studies in relation to subject content and research methodology and identifies striking deficits. Additionally it is also argued, that the implicit didactical approach is obsolete. In the final section, the paper discusses the potential of key concepts for the improvement of the integrated subject of geography and economic education.

Keywords: economic education, empirical studies, learning theories, curriculum making, key concepts

Der GW-Unterricht stellt ein österreichisches Spezifikum dar. Zwar ist die Geographie in anderen Ländern ebenfalls oft mit anderen Fächern gekoppelt oder weist zumindest einen Schwerpunkt in den Natur-, Sozial- oder Humanwissenschaften auf, aber nirgends sonst ist sie mit dem Fach Wirtschaft verbunden (vgl. Uhlenwinkel et al. 2016). Zwar ist die Wirtschaft selten als eigenständiges Fach konzipiert, aber die Verbünde, in denen sie sich findet, bestehen meist aus einer Kombination mit entweder den sozialwissenschaftlichen Fächern oder sie bilden ein arbeitsorientiertes Integrationsfach, oftmals kombiniert mit Arbeitslehre (vgl. Hedtke 2015). Eher selten haben diese Verbindungen so lange Bestand gehabt wie die Verbindung von „G“ und „W“ in Österreich. In jüngster Zeit wird diese Verbindung von Seiten der Wirtschaftsverbände sowie von Teilen der Wirtschaftspädagogik allerdings massiv in Frage gestellt. Die Kritik reicht dabei von der tendenziell eher populistischen Feststellung, dass die Finanzbranche aus der Bankenkrise ab 2007 unter anderem gelernt habe, dass die „Finanzausbildung an österreichischen Schulen ,eine Katastrophe“" sei (Kleine Zeitung 2016, Finanzen 2016), über Studien zu Schulbüchern, die zeigen sollen, dass der GW-Unterricht fehlerhaft sei und das Unternehmertum zu negativ darstelle (vgl. Haigner et al. 2016), bis zu Untersuchungen zum Wissen der Schüler/innen, welche die Effektivität des GW-Unterrichts in Frage stellen wollen (vgl. Rumpold \& Greimel-Fuhrmann 2016).

Im Zentrum des vorliegenden Beitrags steht eine inhaltliche und methodische Auseinandersetzung mit der in den empirischen Studien aus der Wirtschaft und Teilen der Wirtschaftspädagogik vorgebrachten Kritik. Nicht intendiert ist eine Gegenüberstellung der empirischen Resultate dieser Studien mit Forschungsergebnissen aus der Geographiedidaktik (vgl. Fridrich 2015). Ebenso wenig soll, die Realität' als Bezugspunkt herangezogen werden, da hier von der Unmöglichkeit der Überprüfung von Wahrnehmung an 
der nicht wahrgenommenen Welt ausgegangen wird (vgl. Goodman 1984). Nichtsdestotrotz finden sich Verweise auf die Schulpraxis, indem die Diskussion der Kritik von einem vorgeschalteten kurzen Rückblick auf die Entwicklung des GW-Unterrichts und nachgeschalteten Überlegungen zur Weiterentwicklung des Faches ,Geographie und Wirtschaftskunde auf Basis der jüngsten curricularen Gestaltung des Faches (vgl. Pichler 2014, BMB 2016) eingerahmt wird.

\section{Eckpunkte der Entwicklung des GW-Unterrichts}

Die folgende Darstellung der Entwicklung des GWUnterrichts erhebt keinen Anspruch auf Vollständigkeit. Derartige Darstellungen sind bereits an anderer Stelle publiziert worden (vgl. Sitte, C. 1989, Sitte, W. 2001). Stattdessen sollen hier nur einige Eckpunkte benannt werden, welche für die Einordnung und Diskussion der oben angeführten Studien zur Behandlung der Wirtschaft im GW-Unterricht relevant erscheinen. Als heuristisches Modell werden dabei die drei Szenarien von Young \& Muller (2010) zugrunde gelegt, da sie einerseits einen allgemeinen Rahmen bieten, der zwar in verschiedenen nationalen Kontexten unterschiedlich gefüllt wird, es aber nichtsdestotrotz erlaubt, theoretisch klassifizierbare Entwicklungen zu erkennen, und sie andererseits genau aus diesem Grund helfen, sowohl die Entwicklungen als auch etwaige Inkompatibilitäten von Ansätzen zu verstehen. Im Hinblick auf die Genese des GW-Unterrichts lassen sich rückblickend drei Phasen unterscheiden:

1. Bereits 1962 beschloss das österreichische Parlament die Bereiche Wirtschaft sowie Gesellschaft aufgrund der zunehmenden Ökonomisierung und Demokratisierung als Teil der Allgemeinbildung anzusehen. Um keine neuen Fächer schaffen zu müssen, wurden diese Bereiche zum einen der Geographie (Wirtschaft) und zum anderen der Geschichte (Sozialkunde) zugeordnet. In der Geographie traf die Wirtschaft auf eine Disziplin, die den Wandel hin zu einer modernen Wissenschaft erst noch bewältigen musste. Entsprechend orientierte sich auch das Schulfach vor allem daran, Wissen über Länder und Landschaften zu vermitteln. Dieser Form der Wissensvermittlung wurden wirtschaftliche Daten und Fakten quasi angehängt (vgl. Sitte, W. 2001). In der Heuristik von Young und Muller (2010) würde dieser Unterricht einem F1-Curriculum entsprechen. Ein solches F1-Curriculum zeichnet sich durch ein weitgehend stabiles, gut umgrenztes Wissen aus, welches die Vorstellungen der herrschenden Klassen reproduziert und Jugendliche aus bildungsferneren Schichten systematisch benachteiligt. In der Geographie kann ein solches Wissen sowohl länderkundlich als auch allgemeingeographisch orientiert sein, sodass auch die frühen Versuche der österreichischen Curriculum-Entwicklung, insbesondere in der Sekundarstufe I, Länder durch Themen zu ersetzen (vgl. Sitte, W. 2001), an dieser Zuordnung zu einem F1-Curriculum zunächst wenig änderten.

2. Mitte der 1980er Jahre erfolgte in Österreich, wie auch in vielen anderen Ländern, eine Transformation hin zu einem F2-Curriculum, in dem Wissen entdifferenziert wird und der Schwerpunkt von speziellen Kenntnissen zu allgemeinen Fähigkeiten und Fertigkeiten wechselt (vgl. Young \& Muller 2010). Im Kern sollten somit eher die Fähigkeit, Entscheidungen zu treffen, oder die Fähigkeit, Machtinteressen zu identifizieren, vermittelt werden als ein bestimmtes fachliches Wissen. Damit einher ging eine Betonung der Interdisziplinarität, da davon ausgegangen wurde, dass viele Fähigkeiten und Fertigkeiten unabhängig von bestimmten Fächern erworben werden können. Fachgrenzen wurden dementsprechend immer weniger relevant und mit der Zeit immer weiter aufgelöst. Diese Neuorientierung erlaubte es dem Integrationsfach GW, Geographie und Wirtschaft als zwei gleichberechtigte Teile eines Faches aufzufassen, die unter der generischen Maxime allgemeiner gesellschaftlicher Handlungskompetenzen, wie Methoden- oder Gesellschaftskompetenz, gemeinsam zum Gelingen von Unterricht beitragen konnten. Faktenwissen und Fachgrenzen verloren dabei an Bedeutung (vgl. Sitte, W. 2001).

3. Das F3-Curriculum kritisiert sowohl das zu wenig gesellschaftsbezogene Wissen des F1-Curriculums als auch das zu stark gesellschaftsbezogene Wissen des F2-Curriculums. Es betont die Relevanz von Fächern als spezialisierten Gemeinschaften der Wissensentwicklung, wobei das entstandene Wissen weder stabil noch undifferenziert ist, sondern konzeptuell und immer nur das zu einem bestimmten Zeitpunkt beste Wissen, das gerade vorhanden ist (vgl. Young \& Muller 2010). Im neuen, semestrierten österreichischen Lehrplan der Sekundarstufe II der AHS lassen sich Aspekte dieses Ansatzes insbesondere in der Ausweisung von Basiskonzepten finden (vgl. Hinsch et al. 2014). Andere Aspekte, wie etwa die Reformulierung von Lernzielen zu Kompetenzen (vgl. Pichler 2014), entsprechen hingegen eher einer sehr eigenen Mischung der Ansätze eines F1- und eines F2-Curriculums. 
Hinsichtlich der folgenden Auseinandersetzung mit den kritischen Einwänden von Vertreterinnen und Vertretern der Wirtschaft sei noch darauf hingewiesen, dass die kompetenzorientierten Ansätze der Nach-PISA-Zeit ihren Ursprung vor allem im neoliberalen Effizienzstreben und dem damit verbundenen Wunsch nach universeller Operationalisierbarkeit haben (vgl. Crouch 2015).

\section{Kritische Betrachtung der Einwände von Vertreterinnen und Vertretern der Wirtschaft}

Bereits nach knapp drei Monaten der Beobachtung von Debatten des deutschen Bundestages stellte Roger Willemsen (2015) fest: „Debatten, von denen große Konzerne betroffen sind, werden meist heftiger geführt als Debatten, in denen bloß immaterielle Werte zur Diskussion stehen. Die Freiheit des Menschen hat konziliantere Anhänger als die Freiheit der Marktwirtschaft" (Willemsen 2015: 165). Eine ähnliche Vehemenz lässt sich in den derzeitigen Auseinandersetzungen um die Wirtschaft im GW-Unterricht entdecken. Dabei scheint die Attacke auf das Fach GW von Seiten der Wirtschaft jedenfalls strukturell gut vorbereitet: Zunächst entstanden eine Reihe von Masterarbeiten (vgl. Felzmann 2015, Forstik 2013, Hajek \& Rupp 2016, Kolm 2014, Piterna 2015), die im Großen und Ganzen zur grauen Literatur zu zählen sind, bevor im Laufe des Jahres 2016 ganze Studien (vgl. Haigner et al. 2016, Rumpold \& Greimel-Fuhrmann 2016) in der offiziellen Literatur publiziert wurden. Diese offizielle Literatur wird im Folgenden im Fokus stehen, ohne die graue Literatur jedoch völlig zu vernachlässigen. Letztere wird dabei allerdings lediglich als informative Hintergrundfolie genutzt, ohne dass sie einer eingehenden Kritik unterzogen werden. Der Fokus der kritischen Betrachtung wird sich zum einen auf zwei inhaltliche Aspekte sowie zum anderen auf die Methodik der Untersuchungen beziehen. Abschließend wird eine didaktische Einordnung der beiden Studien vorgenommen.

\subsection{Inhaltliche Aspekte}

Hinsichtlich der inhaltlichen Aspekte ist die Kritik vonseiten der Wirtschaft am GW-Unterricht durchaus zwiespältig. Diese Ambivalenz lässt sich besonders schön an der von Greimel-Fuhrmann betreuten Masterarbeit von Felzmann (2015) ablesen. Die Autorin untersucht die „aktuelle Umsetzung der Wirtschaftskunde in den Schulbüchern der 7. Klasse" (Felzmann 2015, Titelseite). Im quantitativen Teil ihrer Analyse zeigt sie, dass der Anteil der Wirtschaftsthemen in allen drei untersuchten Schulbüchern sehr hoch ist und den Anteil geographischer Themen bei weitem übersteigt (vgl. Tab. 1). Im qualitativen Teil stellt sie fest, dass in den Schulbüchern die Lehrplanvorgaben „nahezu zur Gänze umgesetzt“ (ebd: 90 - das Zitat bezieht sich auf das Lehrbuch Durchblick, kann aber exemplarisch für alle drei Bücher stehen) werden und die Wirtschaftserziehung in alle drei Bände eingeflossen ist, wenn auch „unterschiedlich stark" (ebd.: 100).

Angesichts dieser Ergebnisse erscheint es fraglich, ob der von der Autorin hypothetisch formulierte „Idealfall, nämlich ein eigenes Fach Wirtschaft" (ebd.: 5) überhaupt erforderlich ist. Diese Skepsis gegenüber dieser Forderung wird erhärtet, wenn es im Fazit der Arbeit heißt, „dass für das Fach Geographie und Wirtschaftskunde derzeit sowohl der Aufbau des Lehrplans wie auch die Konzeption der drei Schulbücher gute Voraussetzungen für eine ökonomische Bildung der AHS-SchülerInnen [sic!] schaffen" (ebd.: 112).

Die Befürworter/innen eines eigenen Faches Wirtschaft müssen also andere, nicht-inhaltliche Begründungen vorbringen, um ein integriertes Fach GW abzulehnen. Die Autorin der Masterarbeit versucht es mit dem Hinweis auf die beschränkte Stundenzahl: „Es ist fraglich, wie diese Stoffvielfalt mit nur zwei Wochenstunden in einem Schuljahr den SchülerInnen [sic!] vermittelt werden kann" (ebd.: 113), sodass zu befürchten sei, „dass die Umsetzung des Lehrplans in der Praxis nicht die nötige ökonomische Bildung der AHS-AbsolventInnen [sic!] garantiert" (ebd.). Da dieser Einwand aber nicht nur das Fach Wirtschaft, sondern auch das Fach Geographie beträfe und ohne eine Neukonfiguration der Fächer durch eine schlichte Stundenerhöhung behoben werden könnte, machen sich die offiziellen Publikationen daran, andere Ansatzpunkte für ihre Kritik zu identifizieren. Ob die Argumente dadurch allerdings überzeugender werden, soll im Folgenden geprüft werden.

\begin{tabular}{|l|c|c|c|c|}
\hline Buch & $\begin{array}{c}\text { wirtschaftliche } \\
\text { Kapitel in Seiten }\end{array}$ & $\begin{array}{c}\text { wirtschaftliche } \\
\text { Kapitel in \% }\end{array}$ & $\begin{array}{c}\text { geographische } \\
\text { Kapitel in Seiten }\end{array}$ & $\begin{array}{c}\text { geographische } \\
\text { Kapitel in \% }\end{array}$ \\
\hline Durchblick 7 & 160 & 75 & 58 & 25 \\
\hline Meridiane 7 & 165 & 65 & 90 & 35 \\
\hline Geospots 7/8 & 95 & 68 & 45 & 32 \\
\hline
\end{tabular}

Tab. 1: Anteile von Wirtschaft und Geographie in ausgewäblten Schulbüchern der 7. Klasse (Felzmann 2015: 76) 


\subsubsection{Darstellung des Unternehmertums}

Eines der beiden von Haigner et al. (2016) vorgebrachten Hauptargumente gegen den GW-Unterricht in seiner aktuellen Form ist, dass in den Schulbüchern ein ,undifferenzierte[r] unternehmenskritische[r] Ton [...] angeschlagen“ (ebd.: 106) sowie „die Macht der Unternehmen und Konzerne stark überbetont" (Stronach in ebd.: 6) werde. Dies sei ein Indikator einer „ideologischen [...] Grundorientierung der Autorinnen und Autoren" (Haigner et al. 2016: 22), welche für die Entwicklung Österreichs geradezu gefährlich sei, weil „Rahmenbedingungen [fehlen], die Unternehmertum fördern [und den] Menschen positive Anreize bieten, sich selbstständig zu machen, Firmen zu gründen, ihre Ideen umzusetzen oder neue Produkte zu entwickeln" (Stronach in ebd.: 5). Der Versuch einer Begründung dieser These wird im Kapitel „Schulbücher zeichnen ihre eigenen Weltbilder“ (ebd.: 29) unternommen, wobei auf den 65 Seiten allerdings lediglich drei Aspekte zu finden sind, welche sich überhaupt mit Unternehmertum beschäftigen:

1. Umweltschutzthemen würden in „Zusammenhang mit multinationalen Unternehmen oder Industrieunternehmen" (ebd.: 42) behandelt. Die Multis würden in diesem Kontext als profitgierige (vgl. ebd.: 27) und machtbesessene Unternehmen dargestellt, die bestenfalls von NGOs, nicht aber von Regierungen gestoppt werden könnten (vgl. ebd.: 92).

2. Das Problem des Wettbewerbs werde an der Verdrängung heimischer Bekleidungsgeschäfte durch international agierende Discounter dargestellt (vgl. ebd.: $58 \mathrm{f}$.).

3. Die Produktion in Niedriglohnländern erscheine als rein profitorientiert und negativ für heimische Arbeitnehmerinnen und Arbeitnehmer sowie für die Umwelt (ebd.: 63f.). Dabei werde jedoch übersehen, dass der Anteil der Lohnkosten am Verkaufspreis beispielsweise eines T-Shirts äußerst gering sei (vgl. ebd.: 59).

Gefragt werden muss in diesem Kontext zunächst nach dem zugrundeliegenden Begriff des „Unternehmertums“. Bereits 1970 unterscheidet der amerikanische Wirtschaftswissenschaftler Milton Friedman in einem Beitrag für die New York Times zwei grundlegend verschiedene Typen von Unternehmern bzw. Unternehmen. Ihm zufolge gibt es auf der einen Seite den Unternehmer, der sein Unternehmen besitzt und führt. Auf der anderen Seite gibt es Unternehmen (corporations), bei denen es sich letztlich um juristische Personen handelt, die von einer Vielzahl von Anteilseignern oder Gesellschaftern besessen werden und deren Manager oder Geschäftsführer regelmäßig Ange- stellte des Unternehmens sind (vgl. Friedman 1970). Diese Manager verfügen somit gerade nicht über das klassische Merkmal eines Unternehmers, nämlich nicht lohn- oder gehaltsabhängig zu sein (vgl. Bögenhold 2017). Die zuvor charakterisierten corporations sind es, die - auch laut Friedman - am ehesten in der öffentlichen Kritik stehen. Dies lässt sich dadurch begründen, dass der Begriff der Verantwortung für den Unternehmer als natürliche Person einerseits und für das Unternehmen als juristische Person andererseits unterschiedlich bestimmt ist. Das Unternehmen als juristische Person könne per se keine gesellschaftliche, sondern eben nur eine juristische Verantwortung übernehmen. Der angestellte Manager dieses Unternehmens ist, jedenfalls in der Zeit, während der er für das Unternehmen arbeitet, ausschließlich den Anteilseignern verantwortlich und hat somit für die Profitabilität des Unternehmens zu sorgen. Lediglich in seiner Freizeit kann er gesellschaftliche Verantwortung übernehmen, da er seine Anliegen dann mit seinen eigenen finanziellen und zeitlichen Ressourcen unterstützt und nicht mit dem Geld und der Zeit seiner Arbeitgeber. Anders sieht es Friedman zufolge beim Unternehmer als natürliche Person aus. Zwar kann auch hier die Übernahme von gesellschaftlicher Verantwortung auf Kosten des Unternehmens gehen, da das Unternehmen aber dem handelnden Unternehmer gehört, kann er auch entscheiden, ob er etwa auf einen Teil seines Gewinns zugunsten gesellschaftlicher Zwecke verzichten möchte (vgl. Friedman 1970). Wird diese differenzierte Betrachtung des Unternehmertums zugrundgelegt, dann kreisen alle drei zuvor genannten Aspekte um Unternehmen als juristische Person, d.h. um die Handlungen von Angestellten. Im zweiten Beispiel ist zudem impliziert, dass das Unternehmen als juristische Person dem Unternehmer als natürliche Person sogar direkt gegenübertritt und damit der angestellte Manager dem Unternehmertum den Boden unter den Füßen wegzieht.

Unterstellt man für einen Moment, dass Haigner et al. (2016) den Begriff des Unternehmertums zwar korrekt verwenden, die Beispiele jedoch unglücklich gewählt haben, ergibt sich als weiteres Problem, dass die Unternehmer/innen als natürliche Personen nicht immer Arbeitnehmer/innen beschäftigen, sondern oftmals Selbstständige in Ein-Personen-Unternehmen sind (vgl. Bögenhold \& Klingmair 2015). In Österreich stellten sie 2013 nahezu $53 \%$ der Unternehmer (vgl. ebd.: 9), womit das Land deutlich unter dem EU(28)-Durchschnitt von gut $71 \%$ liegt. Wenn Haigner et al. (2016) hierin allerdings ein Manko sehen, dann haben sie das nicht getan, was Schülerinnen und Schüler im GW-Unterricht ebenfalls lernen sollen, nämlich internationale Vergleiche anzustellen. Hätten sie dies getan, dann hätten sie feststellen müssen, dass 


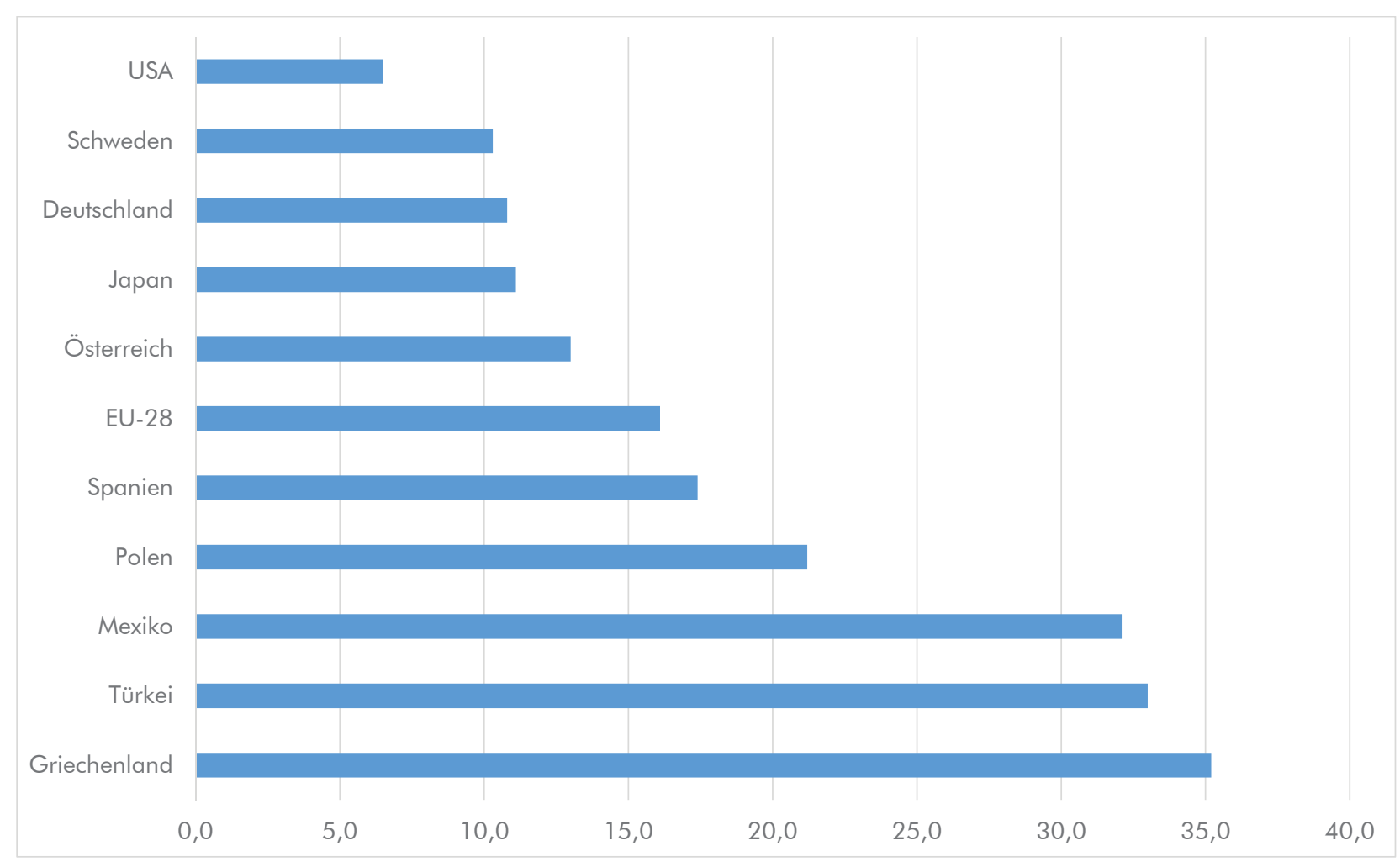

Abb. 1: Anteil der Selbstständigen an allen Erwerbstätigen 2015 (OECD 2018)

der Anteil der Selbstständigen an allen Erwerbstätigen ausgerechnet im Land der Start-Ups, den USA, äußerst gering ist, während er in ökonomisch weniger stabilen Ländern wie beispielsweise in Griechenland oder in einigen Schwellenländern besonders hoch ist (vgl. Abb. 1). Bögenhold (2017) begründet diese Datenlage - konträr zu den impliziten Annahmen der Studie von Haigner et al. (2016) - damit, dass „[ü]berall [dort], wo die Arbeitslosigkeit ansteigt, [...] auch die Selbstständigkeit an[steigt]. Das, was häufig als Medizin gegen die Krankheit Arbeitslosigkeit propagiert wird, ist eigentlich gar keine Medizin, sondern das Symptom" (Bögenhold 2017: 37).

Vermutlich hat der Autor des Vorworts der Schulbuchstudie (Haigner et al. 2016) diesen Zusammenhang nicht gemeint, wenn er sich aufgrund der seit Jahren steigenden Zahl der Arbeitslosen in Österreich Sorgen macht und im nächsten Atemzug die mangelnde Förderung des Unternehmergeists beklagt. Sollte er ihn doch gemeint haben, dann wäre dies zynisch. Sollte er ihn tatsächlich nicht gemeint haben, dann wäre mit Lehner und Stieger (2017) zu konstatieren, dass die Studie letztlich genau das zeigt, was sie bei anderen kritisiert: eine ideologische Grundorientierung (vgl. ebd. 2017: 69).

Da Haigner et al. (2016) das Unternehmertum begrifflich völlig undifferenziert lassen, steht zudem zu befürchten, dass sie den Vorwurf der „einseitigen“ und „verzerrenden“ Darstellung von Unternehmern auf alle oben diskutierten Kategorien von Unternehmertum beziehen: Unternehmer/innen, Manager/innen, Selbstständige - sie alle wären Opfer einer negativen Darstellung in GW-Schulbüchern. Interessanterweise lässt sich diese negative Darstellung aber auch in anderen gesellschaftlichen Kontexten finden. Beispielsweise hat eine Auswertung der Berufe der Mörder in den weitverbreiteten Tatort-Krimis ergeben, dass Unternehmer, Manager und Selbstständige noch vor den Berufskriminellen rangieren (vgl. Abb. 2), wobei die Verfasser der entsprechenden Analyse immerhin auch die Kategorie der Unternehmer i.w.S. ausreichend begrifflich differenziert haben.

Natürlich könnte an dieser Stelle argumentiert werden, dass Schulbücher medialen Darstellungen entgegenwirken sollten. Allerdings geben gerade die realen Manager/innen großer Unternehmen oftmals genug Anlass für die Konstruktion derartiger Charaktere. Jedenfalls gelangen auch Obermayer und Obermaier nach Durchsicht der Panama-Papers zu dem Schluss, dass die deutsche Commerzbank als „Teil der organisierten Kriminalität" (Obermayer \& Obermaier 2016: 46) zu betrachten sei. Und auch die Ermittlungsverfahren gegen jene Mitarbeiter des SiemensKonzerns, die schwarze Kassen eingerichtet hatten, um den lateinamerikanischen Markt zu erschließen (ebd.), deuten auf ein gehöriges Maß krimineller Energie. Soll der Unterricht Schüler/innen in ihrer Lebenswelt und in ihren Alltagsvorstellungen abholen, dann 


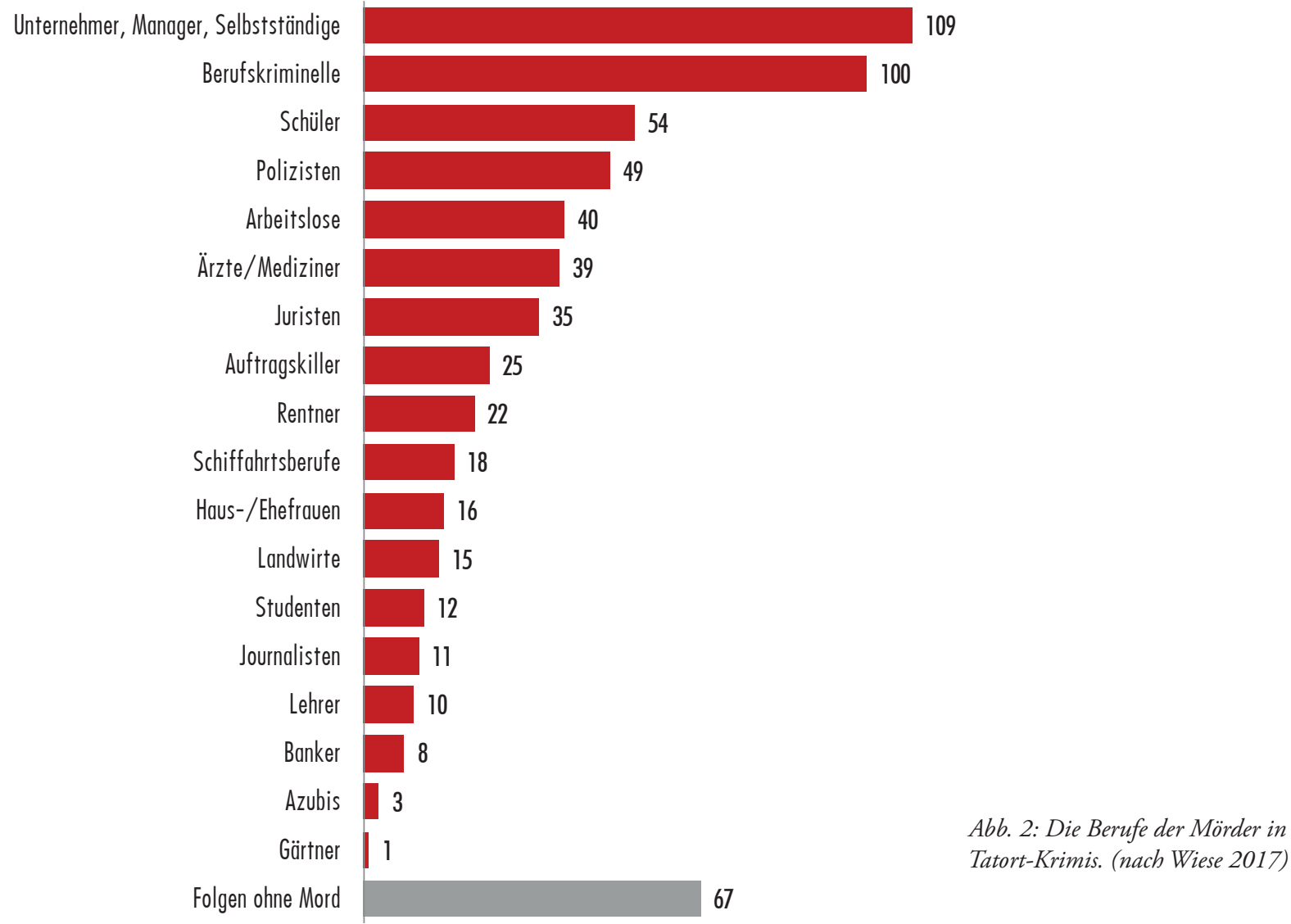

kann und muss an diesen medialen Darstellungen des fiktiven wie des nicht-fiktiven Bereichs zumindest angesetzt werden. Weder die bejahende noch die kritische Darstellung des Unternehmertums ändert aber etwas an der Tatsache, dass das Unternehmersein für viele Selbstständige nicht jenes „Abenteuer“ (Haigner et al. 2016: 97) ist, welches die Autoren unterstellen.

\subsubsection{Input und Output}

Neben der ideologischen Ausrichtung wird von verschiedenen Studien das im GW-Unterricht vermittelte Wirtschaftswissen kritisiert. Während Haigner et al. (2016) ihr Augenmerk dabei auf fehlerhafte Darstellungen in Schulbüchern richten, untersuchen Rumpold und Greimel-Fuhrmann (2016) das bei Schülerinnen und Schülern am Ende der Sekundarstufe I tatsächlich vorhandene Wirtschaftswissen. Die eine Studie konzentriert sich somit auf den Input, die andere hingegen auf den Output.

Hinsichtlich der Ergebnisse der Schulbuchstudie muss zunächst festgestellt werden, dass ein Großteil der als fehlerhaft identifizierten Inhalte sich tatsächlich eher auf geographisches denn auf wirtschaftliches Wissen bezieht. Zwei dieser Beispiele sollen hier herangezogen werden, um die Qualität der Kritik bewerten zu können. Das erste Beispiel thematisiert die Bevölkerungszahl Australiens, das zweite die Definition des primären Sektors. Im Kern behandeln beide
Beispiele Definitionsfragen, wobei das erste Beispiel um die Frage der Reliabilität von statistisch erhobenen Daten ergänzt wird.

$\mathrm{Zu}$ Definitionen bemerkt der Betriebswirtschaftler Kornmeier (2012) in seiner Einführung in das wissenschaftliche Schreiben, dass sie weder wahr noch falsch sein können, sondern bestenfalls zweckmäßig oder unzweckmäßig. Ihre wesentliche Aufgabe liegt darin, sicherzustellen, dass alle Beteiligten unter einem Begriff das Gleiche verstehen. Dabei können sie nie vollständig sein, da jede Definition wiederum Begriffe nutzt, die definiert werden müssten. Wollte man Definitionen bis zum Ende durchführen, drohe „ein sog. Infiniter Regress“ (ebd.: 113). Das gilt nicht nur für die Wissenschaft, sondern erst recht für Schulbücher. Dementsprechend abstrus erscheinen die Monita von Haigner et al. an den Sachdarstellungen in den von ihnen untersuchten Schulbüchern.

Zur Bevölkerung Australiens stellen die Autoren bei Durchsicht der Bücher fest, dass „'Geografie für alle $1^{\prime}$ davon [spricht], dass in Australien rund 21 Millionen Menschen leben, unterwegs 1' aber von 23 Millionen Einwohnerinnen und Einwohnern" (Haigner et al. 2016: 32). Nachdem gemutmaßt wurde, dass es sich hier eventuell um verschiedene Jahre handeln könnte, widmen sich die Autoren am Beispiel eines weiteren Schulbuchs der Frage, ob mit Australien das Land oder der Kontinent gemeint sei und sich die unterschiedlichen Daten somit auf unterschiedliche 
Gebiete beziehen könnten. Diese Vermutung wird von ihnen allerdings verworfen, weil jedes der beiden Schulbücher eindeutig den „Kontinent" benennt (ebd.: 33). Dies führt die Autoren weiter zu Schulbüchern der 4. Klasse, die diesen Fehler scheinbar weitertragen: „Denn in ,Mehrfach Geografie 4' steht, dass der Kontinent Australien und Ozeanien 38 Millionen Einwohnerinnen und Einwohner hat, in ,weltweit $4^{\circ}$ unter der Kapitelüberschrift ,Australien und Ozeanien ' aber wieder von 23,05 Millionen die Rede ist" (ebd.: 34). Dabei bleibt von den Autoren unbemerkt, dass hier eine andere Bezeichnung des Kontinents genutzt wird, die gegebenenfalls auf eine andere Abgrenzung verweisen kann, denn Kontinente sind anders als Staaten nicht durch eindeutige, notfalls kriegerisch zu verteidigende Grenzen gekennzeichnet, sondern wissenschaftliche Konstrukte, über deren Grenzen und Zusammengehörigkeit mit Worten gestritten wird (vgl. Schultz 2013). Da Kontinente keine politisch verwalteten Einheiten darstellen, verfügen sie auch über keine amtliche Statistik, was die Bestimmung u. a. von Bevölkerungszahlen erschwert. Vielleicht ist dieser Umstand ein Grund dafür, warum die Autoren nur die Daten verschiedener Schulbücher vergleichen, nicht aber in die Statistik schauen, die den Darstellungen eigentlich zugrunde liegen müsste. So verzeichnet etwa Wikipedia unter Rückgriff auf das Australian Bureau of Statistics für Ende 2016 eine Einwohnerzahl von 24.314.833 für den Staat Australien. Legt man das Bevölkerungswachstum des Landes zugrunde lägen die Zahlen der meisten zitierten Schulbücher im Rahmen des Möglichen, wenn der Staat Australien gemeint wäre. Wenn der Kontinent Australien gemeint wäre, wird die Sache deutlich schwieriger, weil zunächst geklärt werden müsste, welche Länder dieser Kontinent umfasst. Eine mögliche Definition wäre der Staat Australien zusammen mit der zu ihm gehörigen Insel Tasmanien und der Insel Neuguinea. Damit entstünde eine Bevölkerung von ca. 35,6 Mil- lionen Einwohnerinnen und Einwohnern. Daneben lässt sich der Kontinent Ozeanien definieren, mit dem die pazifische Inselwelt bezeichnet wird. Hier leben ca. 16,5 Millionen Menschen, ein gutes Viertel davon in Neuseeland. Zusammen würden die Kontinente Australien und Ozeanien somit über etwa 52 Millionen Einwohner/innen verfügen.

Zuzustimmen ist dementsprechend dem Fazit der Autoren, dass „Australien [...] offenbar kein leichtes Thema" (Haigner et al. 2016: 34) ist. Allerdings bezieht sich die der Thematik innewohnenden Komplexität nicht auf einzelne Bevölkerungszahlen, sondern vielmehr auf die zutiefst geographische Fragestellung der Abgrenzung von Räumen.

Im zweiten, deutlich wirtschaftlicher orientierten Beispiel wird die Problematik der Definition von den Autoren der Studie selbst aufgegriffen, wenn sie feststellen, dass der primäre Sektor nahezu in jedem Schulbuch anders abgegrenzt wird (vgl. Tab. 2). Allerdings ziehen Haigner et al. (2016) hier nicht den naheliegenden Schluss, dass die unterschiedlichen Zuordnungen auf unterschiedliche konzeptuelle Vorstellungen des primären Sektors zurückzuführen sind, sondern sie orientieren sich einzig und allein an der Definition der Statistik Austria, für die der primäre Sektor sich aus Land- und Forstwirtschaft sowie Fischerei inklusive Jagd zusammensetze (vgl. ebd.). Ein Blick in verschiedene Hand- und Wörterbücher offenbart jedoch, dass auch der Begriff des primären Sektors jedenfalls in seinen Randbereichen nicht unumstritten ist. So definiert das Wörterbuch der Geographie den primären Sektor als denjenigen „Teil der Gesamtwirtschaft, der sich mit der Urproduktion von Rohstoffen befasst. Dazu zählen Landwirtschaft, Forstwirtschaft, Fischerei und der reine Bergbau (ohne Aufbereitung)" (Leser et al. 2001: 649). Diese Definition entspricht den Vorstellungen von Wolfe, der die drei Sektoren nach dem vorherrschenden Produktionsfaktor (Boden, Kapital, Arbeit) kategorisiert (vgl. Pohl 1970).

Tab. 2: Unterschiedliche Definitionen des primären Sektors (Haigner et al. 2016: 51)

\begin{tabular}{|c|c|c|c|c|c|c|}
\hline & Landwirtschaft & Forstwirtschaft & Fischerei & Jagd & Bergbau & $\begin{array}{l}\text { Bergbau ohne } \\
\text { Aufbereitung }\end{array}$ \\
\hline GEOprofil 2 & $x$ & $x$ & $x$ & & & \\
\hline $\begin{array}{l}\text { neugierig auf } \\
\ldots \text { Geographie }\end{array}$ & $x$ & $x$ & $x$ & $x$ & $x$ & \\
\hline Weltreise 2 & $x$ & $x$ & $x$ & & & $x$ \\
\hline $\begin{array}{l}\text { Abenteuer } \\
\text { GW2 }\end{array}$ & $x$ & & $x$ & & $x$ & \\
\hline $\begin{array}{l}\text { Mehrfach } \\
\text { Geografie }\end{array}$ & $x$ & $x$ & & & & \\
\hline $\begin{array}{l}\text { Ganz klar: } \\
\text { Geografie } 2\end{array}$ & $x$ & $x$ & $x$ & $x$ & & \\
\hline
\end{tabular}


Im Online-Wirtschaftslexikon von Gabler umfasst der primäre Sektor „entsprechend des Sektorenschemas von Fourastié [...] die Wirtschaftszweige Landwirtschaft, Forstwirtschaft und Fischerei“" (Springer Fachmedien 2017). Diese Definition nimmt ausdrücklich Bezug auf Fourastié, der die Sektoren nach der Fortschrittlichkeit der eingesetzten Technik (mittel, stark, schwach) einteilt (vgl. Pohl 1970). Die Land- und Forstwirtschaft bildet somit bei beiden Definitionen den Kern des primären Sektors, während die Randbereiche in Abhängigkeit von der gewählten Perspektive unterschiedlich bestimmt werden: In der geographischen Definition geschieht dies ausschließlich über den vorherrschenden Produktionsfaktor, in der wirtschaftlichen Definition hingegen über den Grad der Technikentwicklung.

Bei beiden Beispielen scheint die Kritik der Autoren vor allem darauf abzuzielen, dass die verschiedenen Schulbücher nicht mit exakt denselben Daten und Definitionen operieren. Ein derartiges Ideal des für alle gleichen Unterrichts gab es im deutschsprachigen Raum zuletzt in der DDR (vgl. Budke 2010), wo alle Schüler/innen eines Jahrgangs in derselben Woche und zur selben Zeit denselben Stoff durchnehmen sollten. In Westdeutschland ist dagegen schon in den 1970er Jahren der Beutelsbacher Konsens formuliert worden, in dem es u. a. heißt, dass wissenschaftlich oder politisch strittige Themen auch im Unterricht kontrovers dargestellt werden sollten (vgl. Schneider 1999). Eine autoritäre Vereinheitlichung von Perspektiven in einem oder gar in allen Schulbüchern war somit tunlichst zu vermeiden.

Allerdings impliziert allein die Annahme kontroverser Themen bereits einen anderen Wissensbegriff als jenen, dem Haigner et al. (2016) offenkundig zu folgen scheinen. Denn während sich kontroverses Wissen in der Regel durch Differenziertheit, Komplexität und Entwicklungsfähigkeit auszeichnet, beschränkt sich Faktenwissen auf die Feststellung einfacher Tatsachen und Sachverhalte, deren Richtigkeit vergleichsweise leicht überprüfbar ist.

Eine entsprechende Eingrenzung nehmen Rumpold und Greimel-Fuhrmann (2016) in ihrer Studie über das tatsächliche Wirtschaftswissen von Schülerinnen und Schülern vor, wenn sie sich „auf faktisches und konzeptuelles Wissen" (ebd. 2016: 124) konzentrieren und in diesem Rahmen ,vorrangig die Niveaustufen Verstehen und Anwenden" (ebd.) im Blick haben. Begründet wird die so vorgenommene Einschränkung sowohl mit der Angemessenheit dieser Niveaus für Lernende der achten Schulstufe als auch mit den Notwendigkeiten standardisierter Erhebungsmethoden, die ausschließlich richtige oder falsche Antworten zulassen. Da ein Großteil der Darstellungen wirtschaftlicher Zusammenhänge jedoch kontrovers ist und es zumeist sowohl arbeitgeber-als auch arbeitnehmernahe Interpretationen wirtschaftlicher Entwicklung gibt, setzt die Forderung nach eindeutig richtigen oder falschen Antworten voraus, dass die Forschenden sich auf einen klar definierten Ansatz festlegen. Rumpold und Greimel-Fuhrmann fühlen sich dementsprechend der "klassische[n] Wirtschaftstheorie" (ebd.: 125) verpflichtet, ohne diese Wahl näher zu begründen respektive zu diskutieren, wie sie mit Antworten umgehen wollen, die in diesem Kontext zwar falsch, in einem anderen Kontext aber durchaus korrekt wären. Diese Vorgehensweise ist insbesondere deswegen bemerkenswert, weil den $\mathrm{Au}-$ toren durchaus bewusst ist, dass die „klassische Wirtschaftstheorie das wirtschaftliche Handeln der Wirtschaftssubjekte sicherlich nicht restlos erklären kann" (ebd.). Zudem ergibt sich aus didaktischer Perspektive die Anforderung, ,dass trotz notwendiger inhaltlicher Begrenzungen und Vereinfachungen im Sinne der didaktischen Reduktion stets die fachliche Korrektheit der Items gewahrt bleibt" (ebd.: 129). Wie problematisch diese Anforderung letztlich ist, zeigt ausgerechnet das von den Autoren benannte Beispielitem (vgl. Abb. 3), in dem danach gefragt wird, welche Situationen zu einem höheren Wirtschaftswachstum führen. Richtig laut Rumpold und Greimel-Fuhrmann (2016) sind die Antworten 2 und 4, während die anderen beiden Antworten als falsch gewertet werden. Dies erstaunt, zeigt doch fast jede klassische Darstellung des Wirtschaftskreislaufs (vgl. Edling 2010), wie Banken Ersparnisse in Kapital transformieren, um mit

Das Wirtschaftswachstum ist eine wichtige Kennzahl für die wirtschaftliche Entwicklung eines Landes. Welche der folgenden Situationen werden zu einem höheren Wirtschaftswachstum führen? Kreuze diese an! Mehrere Antworten können korrekt sein.

Die Bevölkerung legt mehr Geld auf das Sparbuch, anstatt es auszugeben.

凶 Der Staat lässt neve Häuser und Straßen bauen.

Der Staat erhöht die Steuern und zahlt damit seine Staatsschulden zurück.

凶 Die Unternehmen exportieren mehr Produkte in das Ausland. 
den Gewinnen die anfallenden Zinsen zu finanzieren, d. h. „das Finanzsystem im Allgemeinen, und die Geschäftsbanken im Speziellen, nehmen Ersparnisse entgegen und leiten diese beispielsweise als Kredit weiter" (Herger 2016: 5). Antwort 1 müsste somit zumindest für einen regulären, nicht von einer Nullzinspolitik betroffenen Geldmarkt ebenfalls akzeptiert werden. Weshalb die Autoren die Antwort jedoch nicht als korrekt einstufen, wird von ihnen nicht begründet.

Damit jedoch stellt sich die Frage nach der fachlichen Korrektheit neu, weil unklar bleibt, wann und weshalb eine Antwort als, korrekt' und wann als ,inkorrekt' bewertet wird. Neben diesen in der Regel als qualitativ betrachteten, inhaltlichen Aspekten erweist sich allerdings auch die auf dieser qualitativen Schieflage beruhende methodische quantitative Herangehensweise als problematisch, wie im nächsten $\mathrm{Ab}-$ schnitt zu zeigen sein wird.

\subsection{Methodische Aspekte}

Von der Forschungsmethodik her könnten die den beiden hier diskutierten Untersuchungen zugrundeliegenden Herangehensweisen kaum unterschiedlicher sein. Während Haigner et al. freimütig bekennen, dass es sich bei ihren Ergebnissen lediglich um ,anekdotische[...] Darstellungen“ (ebd. 2016: 96) handelt, da ein „umfassender ,Fakten-Check' nicht Gegenstand“ (ebd.) der Arbeit sei, legen Rumpold und Greimel-Fuhrmann (2106) großen Wert auf ein ausgefeiltes methodisches Instrumentarium, das von einer explorativen Befragung (vgl. ebd.: 128) über eine Pilotierung mittels lautem Denken bis hin zur psychometrischen Itemanalyse (vgl. ebd.: 131), sowohl auf Basis der klassischen als auch der probabilistischen Testtheorie (vgl. ebd.: 132), reicht.

Auch wenn die Frage nach der Forschungsmethodik bei Haigner et al. (2016) letztlich unbeantwortet bleibt (vgl. Lehner \& Stieger 2017), lohnt es doch, die wenigen Hinweise zur Datengrundlage kurz zu reflektieren. Die Autoren haben eigenen Angaben zufolge 57 Schulbücher untersucht, was einem Umfang von rund 6000 Seiten entspricht (vgl. Haigner et al. 2016: 104). Entgegen den Ergebnissen der Studie von Felzmann (2015; vgl. Tab. 1) hat die kursorische Betrachtung des Materials die Autoren zu der Erkenntnis geführt, dass auf diesen Seiten ,überwiegend keine wirtschaftlichen Themen zu finden sind" (Haigner et al. 2016: 104). Diese Erkenntnis wird in den oben genannten Beispielen gespiegelt, die - wie bereits im Kap. 2.1.2 dargestellt - weitgehend geographische Inhalte betreffen. Völlig unabhängig davon, ob es sich um eher geographische oder eher wirtschaftliche Themen handelt, wollen die Autoren in Bezug auf die ca. 6000 Seiten und ohne „Anspruch auf Vollständigkeit“ (ebd.) zu erheben, eine ausreichende Anzahl „zu monierende[r] Darstellungen" (ebd.) gefunden haben, um zu dem Schluss zu kommen, dass „ein kritisches Maß" (ebd.) an Mängeln überschritten sei.

Diese angeblich ausreichende Anzahl an fehlerhaften Darstellungen ist in der Studie auf 65 Seiten in epischer Breite, d.h. mit vielen illustrierenden Abbildungen und in Teilen sogar Wiederholungen, dargestellt. In Bezug auf die 6000 untersuchten Seiten machen 65 Seiten fehlerhafter Darstellungen gerade einmal ein gutes Prozent der Seiten aus. Das ist im Vergleich zu den Ergebnissen anderer Schulbuchstudien verschwindend gering. So hat beispielsweise die deutsche Stiftung Warentest bei der Untersuchung von Biologie- und Geschichtsschulbüchern im Jahre 2007 bei den Biologiebüchern im Schnitt auf jeder fünften und in einzelnen Büchern sogar auf jeder dritten Seite Fehler gefunden, d.h. auf 20-30\% der Seiten (vgl. Tänzler 2007). Diese hohe Anzahl an Fehlern ist im Laufe der Debatte um die Studie der Stiftung Warentest zurückgewiesen worden, da nicht alles als falsch angesehen werden kann, was nur Ergebnis unterschiedlicher Definitionen, Perspektiven oder der didaktischen Reduktion ist (vgl. Stöber 2010). Nichtsdestotrotz zeigt sie aber, dass die Autoren der vorliegenden Studie zu keinen Ergebnissen gekommen sind, die nicht entweder schon bekannt gewesen wären (nämlich, dass Fehler nun mal vorkommen) oder die von der Quantität her außerhalb jedes Rahmens lägen. Damit sollen die in den GW-Schulbüchern gefunden fehlerhaften Darstellungen nicht kleingeredet werden. Jeder Fehler ist ein Fehler zu viel. Zurückgewiesen werden muss allerdings die Behauptung, dass hier ein kritischer Zustand jenseits aller VerhältnismäBigkeit erreicht sei. Das lässt sich aus den vorgelegten Daten definitiv nicht ableiten.

Angesichts der fehlenden Forschungsmethodik bei Haigner et al. erscheint das Forschungsdesign der Studie von Rumpold und Greimel-Fuhrmann geradezu elaboriert, was allerdings nicht zur Folge hat, dass die Untersuchung frei von eklatanten methodischen Mängeln ist. Der gravierendste von ihnen ist hierbei eindeutig die nicht gegebene Repräsentativität der jeweiligen Stichprobe. Dies gilt sowohl für die explorative Befragung und die Pilotierung als auch für die Voruntersuchung. Zur Verdeutlichung der Problematik genügt ein Blick auf die verschiedenen Kategorien, denen sich Schüler/innen zuordnen lassen: Da wären zunächst die sozialstatistischen Merkmale wie das Geschlecht, der familiäre Hintergrund, die Schulform und die Schulstufe. Darüber hinaus wären aber auch didaktische Unterscheidungen zu berücksichtigen, etwa die sechs von den Schulstufen unabhängigen kognitiven Erkenntnisstufen der Bloomschen Taxonomie (vgl. Roberts 2003) oder die vier Lernstile von Kolb (1984). Insbesondere für Wirtschaftsthemen 
wären zudem die sieben bei Jugendlichen identifizierbaren Milieu-Gruppen (vgl. Wippermann \& Calmbach 2007) zu beachten, da Jugendliche verschiedener Milieu-Gruppen ein sehr unterschiedliches Interesse an einzelnen Wirtschaftsfragen haben können: So gelten beispielsweise hedonistische Jugendliche als sehr konsumorientiert, während postmoderne Jugendliche gesellschaftlich produzierte Konsumnormen eher hinterfragen. Ergänzend wären hinsichtlich der räumlichen Verteilung der Befragten in Österreich zumindest Stadt-Land-Unterschiede sowie gerade bei Wirtschaftsthemen auch das allgemeine politische Wahlverhalten der Eltern zu berücksichtigen. Aus der Zuordnung zu einer bestimmten Kategorie in auch nur einem dieser Felder lässt sich dabei regelmäßig nicht ableiten, welcher Kategorie die betreffende Person in einem anderen Feld zuzuordnen ist.

Es entsteht somit eine große Diversität, welche sich weder durch die 41 Befragten der explorativen Studie (vgl. Rumpold \& Greimel-Fuhrmann 2016: 128) noch durch die vier (!) Probanden der Pilotierung (vgl. ebd.: 131) noch durch die „432 Schüler/innen aus 16 Schulen in acht Bundesländern" (ebd.: 133) in der Voruntersuchung abdecken lässt. Ungeachtet der Tatsache, dass bei inferenzstatistischen Untersuchungen stets die absolute und nicht die relative Anzahl der Stichprobenelemente von Bedeutung ist, sei darauf hingewiesen, dass für die Voruntersuchung ca. ein Prozent der etwa 1500 NMSen und AHSen in Österreich berücksichtigt wurde. Betreffend die prozentuale Verteilung der Schüler/innen ergibt sich aus den Daten der Statistik Austria (2016), dass bezogen auf die Schultypen NMS und AHS Unterstufe 56,8\% der Lernenden eine NMS und 43,2\% eine AHS besuchen. Die an der Befragung beteiligten Schüler/innen kamen jedoch zu 50,7\% von der AHS und zu 49,3\% von der NMS (vgl. Rumpold \& Greimel-Fuhrmann 2016: 136). Rumpold und Greimel-Fuhrmann geben mit ihrer Stichprobenauswahl ein Lehrbeispiel $\mathrm{ab}$, wie geschichtete Stichproben nicht gezogen werden sollten, weshalb auch die Aussagekraft der erhaltenen Resultate in Zweifel gezogen werden sollten. Hinsichtlich der Verteilung der Schüler/innen auf die Bundesländer sind zwar abgesehen von Salzburg alle Länder vertreten, allerdings entsprechen die jeweiligen Anteile nicht der allgemeinen räumlichen Verteilung der Schüler/innenzahlen in Österreich (vgl. Tab. 3), womit sich auch hier die Frage nach der Aussagekraft der Ergebnisse stellt. Gerade, weil gesellschaftliche Phänomene regelmäßig sehr komplex sind, hätte es sich angeboten, nur eine zuvor genau definierte Gruppe zu untersuchen, statt die gesamte Population einer Altersgruppe in einer einzigen Studie erfassen zu wollen. Zwar wären die Ergebnisse dann vermutlich nicht so spektakulär, aber sie wären wenigstens valide.
Tab. 3: Probandinnen und Probanden und Schüler/innenzahlen nach Bundesländern (Rumpold \& Greimel-Fuhrmann 2016: 136 sowie eigene Berechnungen auf der Basis von Daten der Statistik Austria)

\begin{tabular}{|l|r|r|r|r|}
\hline & \multicolumn{2}{|c|}{ Probanden } & \multicolumn{2}{c|}{$\begin{array}{c}\text { Schüler gesamt } \\
(2015 / 16)\end{array}$} \\
\hline & Anzahl & $\%$ & Anzahl & $\%$ \\
\hline Wien & 39 & 9,0 & 217.524 & 20,0 \\
\hline Burgenland & 41 & 9,5 & 33.818 & 3,1 \\
\hline Niederösterreich & 135 & 31,3 & 201.423 & 18,6 \\
\hline Oberösterreich & 15 & 3,5 & 193.830 & 17,8 \\
\hline Steiermark & 63 & 14,6 & 144.890 & 13,3 \\
\hline Kärnten & 60 & 13,9 & 69.978 & 6,4 \\
\hline Tirol & 35 & 8,1 & 94.984 & 8,7 \\
\hline Vorarlberg & 44 & 10,2 & 53.931 & 5,0 \\
\hline Salzburg & 0 & 0 & 75.447 & 7,0 \\
\hline
\end{tabular}

Zudem legen Rumpold und Greimel-Fuhrmann (2016) zwar dar, dass Fragebögen aufgrund „offensichtlich unmotivierter oder willkürlicher Bearbeitung" nicht berücksichtigt wurden, machen aber - absichtlich oder unabsichtlich sei dahingestellt - keine Angaben dazu, um wie viele Fragebögen es sich hierbei handelt und ob eine regionale Häufung oder eine aufgrund sozialstatistischer Merkmale auftritt. Offenkundig ist auch der Umgang der Autoren mit den Fragebögen nicht immer stringent, was u. a. daran zu erkennen ist, dass eine Erläuterung des Umgangs mit missing values fehlt. So ergeben in der oben in Teilen zitierten Tabelle (ebd.: 136) die Summen über die Merkmale Alter und Geschlecht 427, über die Merkmale Schultyp und Bundesland 432 und über das Merkmal der in der Familie vorwiegend gesprochenen Sprache 426. Da laut Aussage der Autoren nicht alle Fragebögen berücksichtigt wurden, müssen zumindest die Angaben betreffend der Merkmale Schultyp und Bundesland fehlerhaft sein, weshalb auch an dieser Stelle die Frage gestellt werden muss, welche Aussagekraft die von Rumpold und Greimel-Fuhrmann (2016) aus den Daten gezogenen Schlüsse überhaupt haben.

\subsection{Didaktische Einordnung}

Eine explizite didaktische Einordnung ihrer Untersuchungen wird von den Autorinnen und Autoren der beiden Studien nicht vorgenommen. Implizit lassen sich aber sowohl lern- als auch curriculumtheoretische Grundlagen erkennen, wie im Folgenden gezeigt werden soll.

Hinsichtlich der lerntheoretischen Ansätze lässt sich in der Nachkriegszeit ein Wandel von zunächst eher behavioristisch geprägten Ansätzen hin zu konstruktivistisch geprägten erkennen. Die behavioristische Lerntheorie ging von der Prämisse aus, dass 
ein bestimmter (Lehr-)Input nahezu automatisch zu einem entsprechenden (Lern-)Output führe. Grundlage für diese Annahme war die Vorstellung, dass Dinge aus der Umgebung über die Sinnesorgane wahrgenommen und im menschlichen Gehirn mehr oder weniger realitätsnah abgebildet werden. Diese Prämisse ließ sich empirisch allerdings nicht bestätigen, da Menschen oft nicht lernen, was gelehrt wird. Die konstruktivistische Lerntheorie bietet für dieses Phänomen eine Erklärung. Sie geht davon aus, dass das Gehirn keinen direkten Kontakt zur Außenwelt hat, da alle Sinneswahrnehmung zunächst in einen allgemeinen neuronalen Code übersetzt und dann in einem selbstreferentiellen Prozess erschlossen werden müssen. Übersetzung und Erschließung erfolgen hierbei keinesfalls eindeutig: Dinge, die nicht an bereits vorhandenes Wissen angeschlossen werden können, werden oftmals nicht wahrgenommen, andere werden zwar auf der Ebene des Alltagswissens, aber nicht wissenschaftlich verstanden. (Lehr-)Input und (Lern-) Output können somit a priori nicht als identisch angenommen werden (vgl. Uhlenwinkel 2012).

Sowohl die Untersuchung von Haigner et al. (2016) als auch jene von Rumpold und GreimelFuhrmann (2016) implizieren ein klassisches InputOutput-Modell, da sie davon ausgehen, dass fehlerfreies Lehrwissen zu korrektem Lernwissen wird. In der Studie von Haigner et al. wird nach Fehlern in Schulbüchern gesucht, da die Autoren im Wissen und in den Einstellungen der Schüler/innen Defizite ausmachen. In der Studie von Rumpold und GreimelFuhrmann (2016) sollen mangelnde Kenntnisse der Schüler/innen als Beleg für ein Defizit in der unterrichtlichen Tätigkeit dienen. Nicht nur aufgrund der fehlerhaften statistisch-methodischen Vorgangsweise, sondern auch wegen der als überholt anzusehenden lerntheoretischen Prämissen, auf denen die beiden Untersuchungen beruhen, kann ihr praktischer Wert mehr als bezweifelt werden.

Im Rahmen der in Abschnitt 1 dargestellten Dreiteilung der Curriculumentwicklung würden die lerntheoretischen Ansätze von Haigner et al. (2016) und Rumpold und Greimel-Fuhrmann (2016) weitgehend mit einem F1-Curriculum in Verbindung gebracht werden müssen. In beiden Studien wird vor allem auf genau definierbares, stabiles Wissen abgehoben, welches sich über Lernprogramme lehren und lernen lässt. Ein den Bedürfnissen der heutigen Welt angepasstes wissenschaftliches Wissen, das den Jugendlichen die Möglichkeit gibt, die sich verändernde Komplexität der Welt selbst zu erschließen, entsteht auf diese Weise nicht. Hier liegt jedoch die derzeitige Herausforderung, weswegen Studien zum Lernerfolg unter anderem auf konzeptuelles Wissen ausgerichtet sein sollten.

\section{Integration von Geographie und Wirt- schaftskunde im Kontext des konzeptuel- len Ansatzes}

Eine konzeptuelle Betrachtung des Lerninhalts wird, wie bereits in der Einleitung dargestellt, in dem neuen semestrierten Lehrplan der Sekundarstufe II der AHS angestrebt. Zum Abschluss dieses Beitrags soll ergänzend gezeigt werden, wie das wissenschaftliche key concept Konsum des dort didaktisch definierten Basiskonzepts ,Arbeit, Produktion und Konsum' im GW-Unterricht genutzt werden kann, um die beiden Fächer Geographie und Wirtschaft sinnvoll zu integrieren. Dabei wird hier lediglich auf die Darstellungen des key concept in einer wirtschaftswissenschaftlichen (vgl. Rutherford 2007) und in einer wirtschaftsgeographischen (vgl. Aoyama et al. 2011) Publikation Bezug genommen, da dieser Zugang genügt, um das grundlegende Argument zu verdeutlichen und umgekehrt eine breitere Diskussion den Umfang dieses Beitrags sprengen würde.

Rutherford (2007) sieht im Konsum das Gegenstück zur Investition mit dem Ziel der Produktion: Dinge werden nicht produziert, sondern verbraucht. Dabei wird in der Mikroökonomie unterstellt, dass der Konsument seinen Nutzen maximieren will. Um errechnen zu können, wann der Konsument wie viel verbraucht, wird das Verhältnis von Grenznutzen und Preisen mittels Gleichungen bestimmt. Diese Gleichungen konnten zunächst nur Quantitäten erfassen, was zu neueren Ansätzen geführt hat, bei denen auch Qualitäten wie beispielsweise Produkteigenschaften und Statuswerte erfasst wurden. In der Makroökonomie werden die Gesamtkonsumtion und das Einkommen in Beziehung gesetzt: Wächst das Einkommen, wächst auch der Konsum, allerdings nicht mit der gleichen Geschwindigkeit. Die Ökonomie sieht Konsum somit vor allem als eine monetäre Größe, die sich in Abhängigkeit von und in Konkurrenz zu anderen Geldgrößen ergibt.

Aoyama et al. (2011) interessieren sich aus wirtschaftsgeographischer Sicht eher für die Art der Distribution der Produkte. Angefangen bei Christaller, der anhand von Fahrtkosten und -zeiten die optimalen Handelsstandorte in einer Fläche zu bestimmen suchte, verschob sich die Aufmerksamkeit zunächst auf die Ausbreitung von großen Handelsunternehmen in der Fläche, ihre Konkurrenz zu kleineren Unternehmen, die sich in verschiedenen Gesellschaften unterschiedlich entwickelte, und ihre Internationalisierung. Mit der durch das Angebot regionaler und globaler Produkte zunehmenden Differenziertheit von Konsumentenentscheidungen gerieten auch die Produktionsketten in den Blick. Ihre Kenntnis sollte es den Konsumenten ermöglichen, Produkte bewusst 
auszuwählen, um dadurch Druck auf die Unternehmen ausüben zu können, Arbeitsbedingungen zu verbessern oder Umweltstandards einzuhalten. Die Geographie beschreibt den Konsum somit vor allem hinsichtlich der Frage, wie Produkt und Konsument räumlich zueinanderfinden.

Beide Ansätze, der ökonomische wie der geographische, haben ihre Stärken und ihre blinden Flecken, wie sich an einem der Befunde aus der Studie von Haigner et al. (2016) belegen lässt. Dort heißt es: „Immer scheint es so zu sein, dass alleine das Angebot für das Marktergebnis verantwortlich zeichnet. Kein Wort davon, dass es auch an den Konsumentinnen und Konsumenten liegt, wenn umweltpolitische Maßnahmen nicht umgesetzt werden können" (Haigner et al 2016: 102). In der ökonomischen Theorie haben die Konsumentinnen und Konsumenten tatsächlich keine Verantwortung für die Umwelt, da sie nur Teil einer Gleichung sind, in welcher Grenznutzen und Preise in Relation zueinander gesetzt werden. Sie sind rein ökonomische Subjekte. Aus der geographischen Perspektive werden die Konsumentinnen und Konsumenten hingegen oftmals mit einem übergreifenden Verantwortungsanspruch konfrontiert, dem sie kaum gerecht werden können. Sie werden vor allem als Staatsbürger betrachtet. Im ökonomischen key concept fehlt der staatsbürgerlich handelnde Konsument, in der neueren geographischen Perspektive dagegen der ökonomisch beschränkte Konsument, dessen Einkommen nicht immer ausreicht, um sich verantwortungsvollen und nachhaltigen Konsum leisten zu können. Das Individuum ist aber stets beides zugleich, nämlich Staatsbürger und ökonomisch handelnder Konsument. Dementsprechend sind gerade in der Schule, deren Aufgabe nicht darin besteht, Wissenschaftler/innen auszubilden, sondern mündige Bürger/innen, beide Perspektiven von Relevanz. Und eine Kombination dieser im Alltag oftmals eng verwobenen Bereiche in einem Schulfach scheint dafür tatsächlich überaus geeignet. Es gibt somit keinen Grund, auf das österreichische Spezifikum Geographie und Wirtschaftskunde zu verzichten. Vielmehr sollte es gestärkt und international bekannter gemacht werden.

\section{$4 \quad$ Fazit}

Die jüngsten Versuche von Seiten verschiedener Vertreter/innen der Wirtschaftsverbände oder Teilen der Wirtschaftspädagogik die Leistungen des Faches GW in Frage zu stellen, erweisen sich als wenig überzeugend. Dies liegt nicht nur an den inhaltlichen sowie methodischen Mängeln der Studien, sondern auch daran, dass sie auf überholten lern- und curriculum- theoretischen Grundlagen beruhen. Ihre Aussagekraft ist somit insgesamt begrenzt.

Dieser Befund bedeutet natürlich nicht, dass sich der GW-Unterricht nicht verbessern ließe. Ein Weg in die richtige Richtung wird durch die Einführung der Basiskonzepte im neuen semestrierten AHS-Oberstufen-Lehrplan beschritten. Diese Konzepte konnten schon aufgrund ihrer Aktualität bisher kaum Eingang in die praktische Unterrichtsarbeit finden. Wie eine Integration der beiden Fächer mithilfe dieser Konzepte aussehen könnte, wurde im letzten Kapitel der vorliegenden Arbeit jedenfalls theoretisch angerissen. Es wäre wünschenswert, wenn sich Geographinnen und Geographen und Wirtschaftswissenschaftler/innen zusammenfinden könnten, die Konzepte mit entsprechendem Leben zu füllen.

\section{Dank}

Ich danke Herrn Gert Wolf für wertvolle Hinweise insbesondere in Bezug auf die in diesem Beitrag diskutierten methodischen Aspekte.

\section{Literatur}

Aoyama, Y., J. T. Murphy \& S. Hanson (2011): Key Concepts in Economic Geography. Los Angeles, London, New Delhi, Singapore, Washington DC: Sage.

BMB - Bundesministerium für Bildung (2016): Lehrplan der AHS-Oberstufe für Geographie und Wirtschaftskunde. In: Bundesgesetzblatt II Nr. 219 am 9. 8. 2016, 59-67. https://www.ris.bka.gv.at/Dokumente/BgblAuth/BGBLA_2016_II_219/BGBLA_2016_II_219. pdf (15.4.2018).

Bögenhold, D. (2017): Die andere Seite der Selbstständigkeit. In: ad astra $1 / 37$.

Bögenhold, D. \& A. Klingmair (2015): Micro-Entrepreneurship: Tendenzen der Präkarisierung auf dem Arbeitsmarkt? Empirische Ergebnisse für Kärnten. https://mpra. ub.uni-muenchen.de/63771/1/MPRA_paper_63771. pdf (7.7.2017).

Budke, A. (2010): Und der Zukunft abgewandt. Ideologische Erziehung im Geographieunterricht der DDR. Göttingen: $V \& R$ unipress.

Crouch, C. (2015): Die bezifferte Welt. Wie die Logik der Finanzmärkte das Wissen bedroht. Berlin: Suhrkamp.

Edling, H. (2010): Volkswirtschaftslehre - Schnell erfasst. Berlin, Heidelberg: Springer.

Felzmann, A. (2015): Beleuchtung der Entwicklung des Faches Geographie und Wirtschaftskunde in österreichischen AHS und Analyse der aktuellen Umsetzung der Wirtschaftskunde in den Schulbüchern der 7. Klasse. Wien: Masterarbeit WU. 
Finanzen.at (Hrsg.) (2016): Treichl - Finanzausbildung an österreichischen Schulen Katastrophe. Finanzen vom 28.10.2016. http://www.finanzen.at/nachrichten/aktien/ Treichl-Finanzausbildung-an-oesterreichischen-SchulenKatastrophe-1001487576 (27.4.2018)

Forstik, M. (2013): Quo vadis allgemeine Wirtschaftsbildung? - Die fachliche Vorbereitung der Geograpie- und Wirtschaftskunde Lehrkräfte auf die Vermittlung ökonomischen Wissens an der AHS-Oberstufe. Wien: Masterarbeit WU.

Fridrich, C. (2015): Ökonomische Bildung im Trägerfach Geographie und Wirtschaftskunde zwischen Lebensweltorientierung und ökonomischen Zugängen. Teilergebnisse einer empirischen Studie. In: Mitteilungen der Österreichischen Geographischen Gesellschaft 157, 277-300.

Friedman, M. (1970): The Social Responsibility of Business is to Increase its Profits. In The New York Times Magazine vom 13. September 1970.

Springer Fachmedien (Hrsg.) (2017) Gabler Wirtschaftslexikon. http://wirtschaftslexikon.gabler.de/Definition/ primaerer-sektor.html (2.7.2017).

Goodman, N. (1984): Weisen der Welterzeugung. Frankfurt am Main: Suhrkamp.

Haigner, S. D., S. Jenewein \& F. Wakolbinger (2016): Stiefkind Wirtschaftskunde. Eine Analyse von Schulbüchern: einseitig, falsch, tendenziös? Wien: Frank\&Frei.

Hajek, B. \& M. Rupp (2016): Weiterentwicklung der ökonomischen Bildung im allgemeinbildenden Schulwesen in Österreich. Einstellungen, Herausforderungen und Bedürfnisse der Lehrenden im Unterrichtsfach Geographie und Wirtschaftskunde aus der Perspektive der Lehrenden an der AHS Ostösterreich. Wien: Masterarbeit WU.

Hedtke, R. (2015): Sozioökonomische Bildung als Innovation durch Tradition. In: GW-Unterricht 140, 18-38.

Herger, N. (2016): Wie funktionieren Zentralbanken? Geld- und Währungspolitik verstehen. Wiesbaden: Springer Gabler.

Hinsch, S., H. Pichler, T. Jekel, L. Keller \& F. Baier (2014): Semestrierter Lehrplan AHS, Sekundarstufe II. Ergebnis der ministeriellen Arbeitsgruppe. In: GW-Unterricht 136, 51-61. http://www.gw-unterricht.at/images/pdf/ gwu_136_51_61_hinsch_pichler_jekel_keller_baier.pdf (15.4.2018).

Kleine Zeitung (2016): Weltspartag bei Erste Bank. Sprinkleranlage ausgelöst: Kalte Dusche für Finanzminister. Kleine Zeitung vom 28.10.2016. http://www.kleinezeitung.at/wirtschaft/5109423/Weltspartag-bei-ErsteBank_Sprinkleranlage-ausgeloest_Kalte (27.4.2018)

Kolb, D. (1984): Experiential Learning. Experience as the Source of Learning and Development. New Jersey: Prentice Hall.

Kolm, S. (2014): Financial Literacy - eine empirische Studie bei Schüler/-innen der Handelsakademie sowie Studierenden der Wirtschaftsuniversität Wien. Wien: Masterarbeit WU.
Kornmeier, M. (2012): Wissenschaftlich schreiben leicht gemacht - für Bachelor, Master und Dissertation. Bern, Stuttgart, Wien: Haupt.

Lehner, M. \& S. Stieger (2017): Buchbesprechung: Team Stronach Akademie (Hrsg., 2016): Stiefkind Wirtschaftskunde. Eine Analyse von Schulbüchern: einseitig, falsch, tendenziös? Eine Analyse von 57 Schulbüchern aus Geographie und Wirtschaftskunde der 5. bis 8. Schulstufe. In: GW-Unterricht 146, 68-69.

Leser, H., H.-D. Haas, T. Mosimann, R. Paesler \& J. Huber-Fröhli, J. (2001): Wörterbuch Allgemeine Geographie. München: Westermann, dtv.

Obermayer, B. \& F. Obermaier (2016): Panama Papers. Die Geschichte einer weltweiten Enthüllung. Köln: Kiepenheuer \& Witsch.

OECD - Organisation for Economic Co-operation and Development (2017): Self-employment rate2016. https://data.oecd.org/ emp/self-employment-rate.htm\#indicator-chart (9.8.2017)

Pichler, H. (2014): Vorläufiger Endbericht der Semestrierungsgruppe Geographie und Wirtschaftskunde AHS. In: GW-Unterricht 136, 47-50.

Piterna, C. (2015): Ökonomische Bildung an Österreichischen AHS aus der Perspektive der Lehrenden. Wien: Masterarbeit WU.

Pohl, H.-J. (1970): Kritik der Drei-Sektoren-Theorie. In: Mitteilungen aus der Arbeitsmarkt- und Berufsforschung 3/4, 313-325.

Roberts, M. (2003): Learning Through Enquiry. Making Sense of Geography in the Key Stage 3 Classroom. Sheffield: Geographical Association.

Rumpold, H. \& B. Greimel-Fuhrmann (2016): Wirtschaftswissen in der Sekundarstufe I. Entwicklung eines Erhebungsinstruments für die Zielgruppe von Schüler/ inne/n der achten Schulstufe. In: Zeitschrift für ökonomische Bildung 5, 119-149.

Rutherford, D. (2007): Economics. The Key Concepts. London, New York: Routledge.

Schneider, H. (1999): Der Beutelsbacher Konsens. In: Mickel, W. W. (Hrsg.): Handbuch zur politischen Bildung. Grundlagen, Methoden, Aktionsformen. Schwalbach/Ts.: Wochenschau Verlag, 171-178.

Schultz, H.-D. (2013): Grenzen. In: Rolfes, M. \& Uhlenwinkel, A. (Hrsg.): Metzler Handbuch 2.0 Geographieunterricht. Ein Leitfaden für Praxis und Ausbildung. Braunschweig: Westermann, 326-332.

Sitte, C. (1989): Entwicklung des Unterrichtsgegenstandes Geographie, Erdkunde, Geographie und Wirtschaftskunde an den allgemeinbildenden Schulen (APS u. AHS) in Österreich nach 1945. Wien. http://homepage.univie. ac.at/christian.sitte/Dissinhalt.htm (2.7.2017).

Sitte, W. (2001): Geographie und Wirtschaftskunde (GW) - Entwickung und Konzept des Unterrichtsfachs. In: Sitte, W. \& H. Wohlschlägl, (Hrsg.): Beiträge zur Didaktik des „Geographie und Wirtschaftskunde“-Unterrichts. Wien: Materialien zur Didaktik der Geographie und Wirtschaftskunde, 157-169. 
Statistik Austria (2016): Bildung in Zahlen 2014/15. Schlüsselindikatoren und Analysen. Wien: Selbstverlag.

Stöber, G. (2010): Schulbuchzulassung in Deutschland. Grundlagen, Verfahrensweisen und Diskussionen. Eckert. Beiträge 2010/3. http://repository.gei. de/bitstream/handle/11428/92/715816195_2016_A. pdf? sequence $=2 \&$ isAllowed $=y$ (2.7.2017).

Tänzler, J.-Y. (2007). Blauer Brief für Schulbuchverlage. https://www.welt.de/wams_print/article1223700/Blauer-Brief-fuer-Schulbuchverlage.html (18.5.2018).

Uhlenwinkel, A. (2012): Binnendifferenzierung. In Haversath, J.-B. (Hrsg.): Geographiedidaktik. Braunschweig: Westermann, 330-343.

Uhlenwinkel, A., T. Béneker, G. Bladh, S. Tani \& D. Lambert (2016): GeoCapabilities - A survey of teachers perspectives in Finland, Germany, The Netherlands and Sweden. In: International Research in Geographical and Environmental Education 26 (4), 327-341. https://www.tandfonline.com/doi/abs/10.1080/103820 46.2016.1262603 (1.5.2018)

Wiese T. (2017): Der Gärtner ist fast nie der Mörder. In: Statista (Hrsg.) Das Statistikportal, Medien \& Marketing, Rundfunk, TV \& Film, Tatort. Hamburg. https://de.statista. com/infografik/9764/moerder-im-tatort-krimi/ (7.7.2017).

Willemsen, R. (2015): Das Hohe Haus. Ein Jahr im Parlament. Frankfurt am Main: Fischer.

Wippermann, C. \& M. Calmbach (2007): SINUS-Milieustudie U 27. Wie ticken Jugendliche? Düsseldorf: Haus Altenberg.

Young, M. \& J. Muller (2010): Three Educational Scenarios for the Future: lessons from the sociology of knowledge. In: European Journal of Education 45 (1), 11-27. 